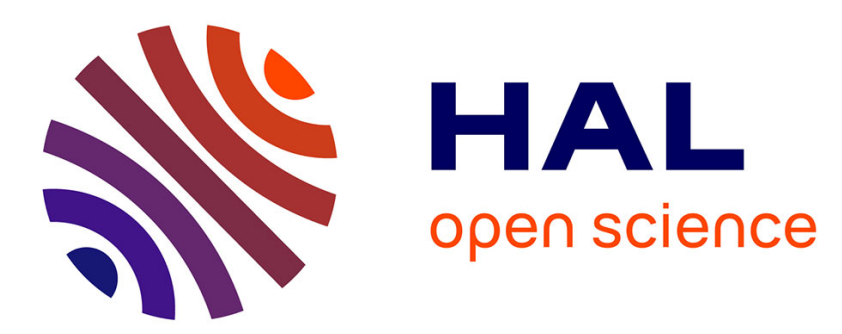

\title{
High-Speed All-Optical Reservoir Computing Using VCSEL Polarization Dynamics
}

Jeremy Vatin, Damien Rontani, Marc Sciamanna

\section{To cite this version:}

Jeremy Vatin, Damien Rontani, Marc Sciamanna. High-Speed All-Optical Reservoir Computing Using VCSEL Polarization Dynamics. 2018 European Conference on Optical Communication (ECOC), Sep 2018, Rome, Italy. pp.1-3, 10.1109/ecoc.2018.8535419 . hal-02002771

\section{HAL Id: hal-02002771 \\ https://hal.science/hal-02002771}

Submitted on 20 May 2020

HAL is a multi-disciplinary open access archive for the deposit and dissemination of scientific research documents, whether they are published or not. The documents may come from teaching and research institutions in France or abroad, or from public or private research centers.
L'archive ouverte pluridisciplinaire HAL, est destinée au dépôt et à la diffusion de documents scientifiques de niveau recherche, publiés ou non, émanant des établissements d'enseignement et de recherche français ou étrangers, des laboratoires publics ou privés. 


\title{
High-Speed All-Optical Reservoir Computing Using VCSEL Polarization Dynamics
}

\author{
Jeremy $\operatorname{Vatin}^{\left(1,2,{ }^{*}\right)}$, Damien Rontani ${ }^{(1,2, \dagger)}$, Marc Sciamanna ${ }^{(1,2 \ddagger)}$ \\ (1) Chair in Photonics, CentraleSupelec and Universite Paris-Saclay, 2 rue Edouard Belin, Metz, 57070 \\ (2) LMOPS EA-4423, CentraleSupelec and Universite Lorraine, 2 rue Edouard Belin, Metz, 57070 \\ ${ }^{*}$ ) jeremy.vatin@centralesupelec.fr

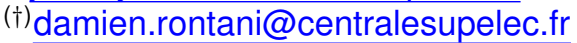 \\ ( marc.sciamanna@centralesupelec.fr
}

Abstract We design a new time-delay reservoir computer based on VCSELs with optical injection. It benefits from the specific VCSEL polarization dynamics in order to process data faster with enhanced performance.

\section{Introduction}

Several thousand gigabits of data are being exchanged each second on the internet network. This ever growing amount of data requires both higher speed and novel processing approach. Beside the already existing solutions, reservoir computing is exploring the possibility to build very efficient, dedicated devices capable of performing different tasks, such as the one required by telecommunication systems (header recognition, channel equalization, etc). Reservoir computing ${ }^{1}$ is part of architecture studied in the field of machine learning. Being inspired by the architecture of the brain, it aims at drawing benefits from interconnected nonlinear systems in order to perform complicated calculation, faster than what a computer is able to do. Reservoir computing can also be split into two categories. One of them is focusing on reservoir composed of several interconnected physical systems. The other one is dealing with time-delay reservoir ${ }^{2}$. Because it is usually very challenging to experimentally connect a large number of physical systems, time-delay reservoir computing uses only one nonlinear system, and then create virtual neurons, distributed along the delay line. Several architectures have been tested, including either electronic ${ }^{2}$, opto-electronic ${ }^{3,4}$ or optical ${ }^{5}$ systems. Here, we propose to study time-delay reservoir computer using a Vertical Cavity Surface Emitting Laser (VCSEL) as a physical system.

VCSELs have several advantages compared to other coherent light sources ${ }^{6}$. Their intrinsic fast modulation bandwidth, low threshold current and single mode operation make them ideal sources for telecommunication application. As a matter of fact, they have replaced edge-emitting lasers in short-haul telecommunication links. These and other advantages, such as rich polarization dynamics, motivate also their use in reservoir computing. We therefore use VCSEL here to design an efficient high-speed reservoir computer (see Fig. 1).

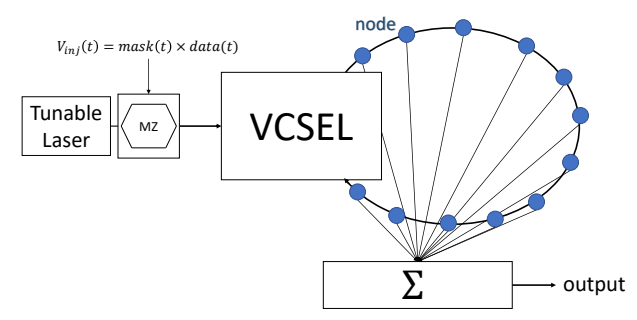

Fig. 1: Principle scheme

The unique physical node of our reservoir is composed of a VCSEL. We are operating a feedback loop on this node, along which we spread the virtual nodes. We can modify the number of virtual nodes $N$, which are separated by a time $\theta$, simply by adjusting the time-delay $\tau=N \theta$. The feedback strength can also be adjusted. The data stream modulates the intensity of a second laser beam which is then optically injected in the VCSEL.

Due to the large number of free parameters in the system, we start by simulating numerically our architecture in order to find the best operating point. This point is chosen in order to allow the reservoir computer to provide its best performance. Two criteria are typically used to unveil the best operating point: the computational ability ${ }^{7}$ and the memory capacity ${ }^{8}$. The first one measures the capacity of a reservoir computer to separate different inputs, and to gather similar ones. This is 
one of the most important property a reservoir should achieve to perform calculation properly. Fig. 2 shows how computational ability varies as a function of the delay loop length.

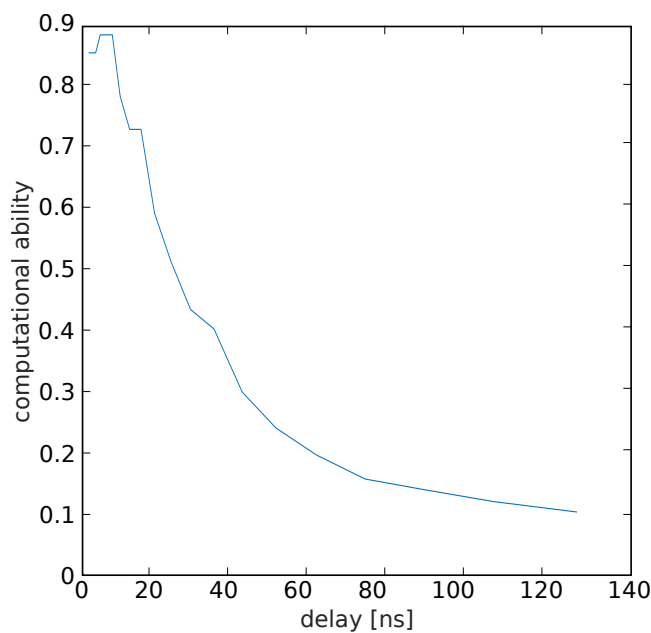

Fig. 2: Computational ability depending on the total delay with 400 virtual nodes. The value has been normalised between 0 and 1 . The higher it gets, the better the system is able to do calculation.

We can notice that a maximum is reached for short delay ( $\leqslant 10 \mathrm{~ns}$ ). This is a huge advantage. Using shorter delay is a way to increase the rate of data processed with time-delay based reservoir computing architecture.

The memory capacity measures the ability of a reservoir computer to remember previous inputs. Some tasks require a high memory capacity because of non-linear transformations, especially while trying to reconstruct signals ${ }^{9}$. Performing such a test with our reservoir allows to get insight into which tasks can be solved using our VCSELbased architecture. Fig. 3 shows results for different sets of parameters.

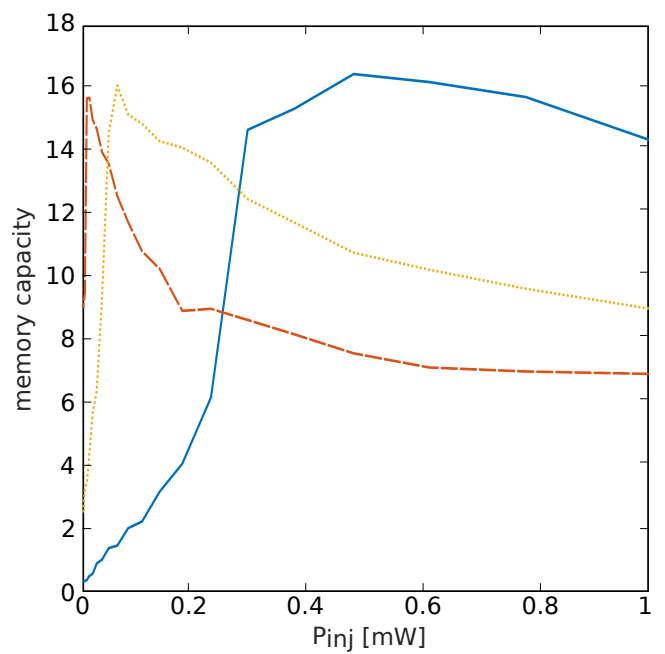

Fig. 3: Memory capacity depending on the injection power for different feedback strength: $16 \mathrm{GHz}$ (dashed line), $32 \mathrm{GHz}$ (dotted line), and $63 \mathrm{GHz}$ (solid line)
With those parameters, the best reachable memory depth is around 16 . This performance can be achieved for multiple combinations of parameters.

Once the reservoir computer is tested, and the operating point is known, we can operate the reservoir computer to solve a particular task. Fig. 4 shows the computational performance on the prediction task of the chaotic time serie Santa Fe. The performance metrics used for this task is the Normalised Mean Square Error (NMSE), defined as follows:

$$
N M S E=\frac{1}{N} \sum_{i=1}^{N}(\hat{y}(i)-y(i))^{2},
$$

where $N$ is the number of samples tested, $y$ the desired output, and $\hat{y}$ the output of the reservoir computer. In the following we shall compare the NMSE performance when accounting or not for the characteristic VCSEL polarisation dynamics.
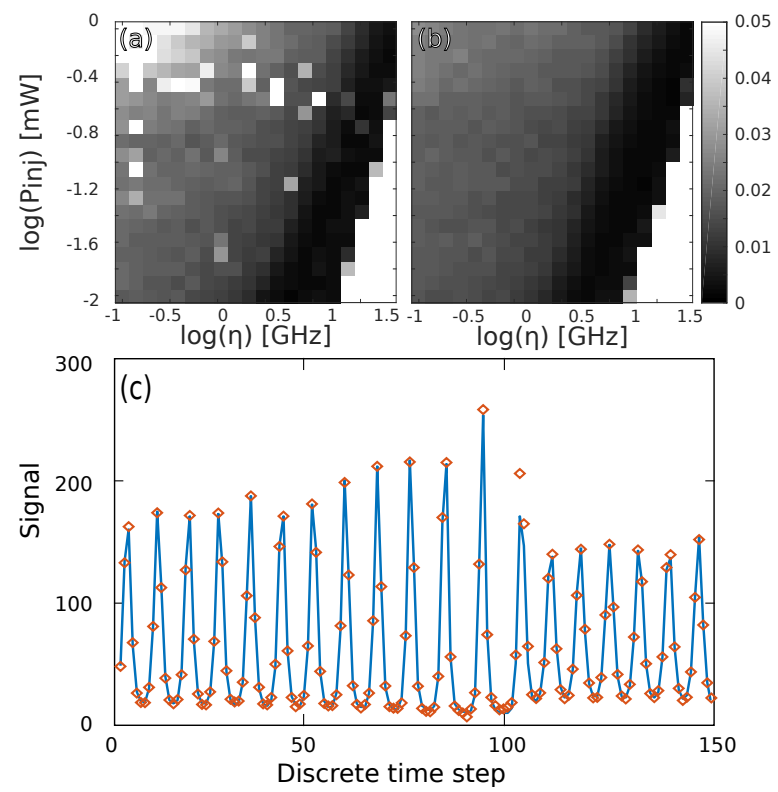

Fig. 4: Santa Fe serie prediction

a) NMSE after prediction for isotropic injection. b) NMSE after prediction for rotated injection.

c) Example of prediction of chaotic signal: the original signal (blue) and the predicted signal (red diamond)

With isotropic feedback, our reservoir computer successfully performs the chaos prediction task with a NMSE of $5 \times 10^{-3}$. In that case the VCSEL is lasing along its main axis only. We then performed the same task with feedback having its polarization rotated. This modification allows to enhance the performance: NMSE now reaches $2 \times 10^{-3}$. Rotating the feedback allows to trigger the two lasing mode of the VCSEL. That seems to be a key to achieve better computational per- 
formance.

We therefore tested other tasks with our device, such as the nonlinear channel equalization. The observation is the same: using a rotated feedback allows to increase the computational performance. We successfully reached an error rate of $2 \times 10^{-5}$ with a rotated feedback, compared to one of $2 \times 10^{-4}$ with isotropic feedback.

\section{Conclusions}

VCSELs are interesting devices thanks to their high speed modulation ability, but also the richness of their polarization dynamics. It appears that we can draw benefits from those properties in reservoir computing to do calculation. The system shows indeed good performances not only in terms of computational ability, but also in terms of memory. That allows to use it on applied tasks, and obtain satisfying performances. These improved properties of reservoir computing have been tested on different tasks such as chaos time series prediction or nonlinear channel equalization.

\section{Acknowledgements}

The authors thank the following founders of the Chaire Photonique: Ministry of High Education and Research, Region Grand Est, Departement of Moselle, European Union (FEDER), Metz Metropole, Airbus GDI Simulation, CentraleSupelec and Fondation Supelec.

\section{References}

[1] Herbert Jaeger and Harald Haas. Harnessing nonlinearity: Predicting chaotic systems and saving energy in wireless communication. Science, 304(5667):78-80, 2004.

[2] L. Appeltant, M. C. Soriano, G. Van der Sande, J. Danckaert, S. Massar, J. Dambre, B. Schrauwen, C. R. Mirasso, and I. Fischer. Information processing using a single dynamical node as complex system. Nature Communications, 2:468 EP -, Sep 2011.

[3] L. Larger, M. C. Soriano, D. Brunner, L. Appeltant, J. M. Gutierrez, L. Pesquera, C. R. Mirasso, and I. Fischer. Photonic information processing beyond turing: an optoelectronic implementation of reservoir computing. Opt. Express, 20(3):3241-3249, Jan 2012.

[4] Yvan Paquot, Francois Duport, Antoneo Smerieri, Joni Dambre, Benjamin Schrauwen, Marc Haelterman, and Serge Massar. Optoelectronic reservoir computing. Scientific reports, 2:287, 2012.

[5] Daniel Brunner, Miguel C Soriano, Claudio R Mirasso, and Ingo Fischer. Parallel photonic information processing at gigabyte per second data rates using transient states. Nature communications, 4:1364, 2013.
[6] M. Muller, W. Hofmann, T. Grundl, M. Horn, P. Wolf, R. D. Nagel, E. Ronneberg, G. Bohm, D. Bimberg, and M. C. Amann. 1550-nm high-speed short-cavity vcsels. IEEE Journal of Selected Topics in Quantum Electronics, 17(5):1158-1166, Sept 2011.

[7] L. Appeltant. Reservoir Computing based on Delay-dynamical Systems. PhD thesis, Vrije Universiteit Brussel and Universitat de les Illes Balears, 2012.

[8] Herbert Jaeger. Tutorial on training recurrent neural networks, covering BPPT, RTRL, EKF and the" echo state network" approach, volume 5. GMD-Forschungszentrum Informationstechnik Bonn, 2002.

[9] Junghsi Lee V. John Mathews. Adaptive algorithms for bilinear filtering, 1994. 